\title{
Scale effects on land loss modeling in the Mississippi River Delta
}

\author{
Nina S.-N. Lam ${ }^{\text {a, }}$, Heng Cai $^{\text {a }}$, Lei Zou ${ }^{\text {b }}$, Kam-biu Liu ${ }^{\text {c }}$ \\ ${ }^{a}$ Department of Environmental Sciences, Louisiana State University, nlam@lsu.edu, hengcail @ gmail.com \\ ${ }^{b}$ Department of Geography, Texas A\&M University, lzou@tamu.edu \\ ${ }^{c}$ Department of Oceanography and Coastal Sciences, Louisiana State University, kliul@lsu.edu \\ * Corresponding author
}

Keywords: neighborhood effects, operational scale, coupled natural-human modeling, variogram analysis,

\begin{abstract}
:
In complex natural-human system modeling, often times a first step is to examine the relationships between a dependent variable and a number of independent variables at their locations. The neighborhood effect, also known as a scale effect, has seldom been considered in the analysis. Previous research has shown that scale effects affect the reliability of analysis results, and rigorous scientific studies should take an extra step to examine the scale effects for more accurate analysis and modeling. However, detecting the neighborhood effects of various variables and then incorporating them into a holistic modeling system posts a serious challenge because of the fundamental difference in properties between variables from the human component (e.g., census data) and variables from the natural component (e.g., landscape properties). Moreover, uncertainties involved in data, data scale, algorithms, and scale of analysis make the findings and the interpretations of the findings unreliable. A major issue of modeling neighborhood effects is the determination of appropriate neighborhood size, also known as the spatial context scale or the operational scale. It has been shown in the literature that neighborhood effects vary with the neighborhood size used to compute the effects. Thus, research on how to determine the neighborhood size that best captures the scale of operation of a phenomenon is very much needed so that we can have more confidence in the modeling results.
\end{abstract}

This study examines the use of variogram in detecting the appropriate neighborhood size of the variables involved in land loss modeling in the Mississippi River Delta. The goal is to find out the best combination of variables and their neighborhood sizes that best explain the variation of land loss patterns in the Deltaic region. The Mississippi River Delta has been suffering substantial land loss during the past several decades. Land loss has been a subject of intense research by many researchers from multiple disciplines, aiming at mitigating the land loss process and its potential damages. However, a majority of land loss projections were derived solely from the natural processes, such as sea level rise, regional subsidence, and reduced sediment flows. Very few studies have incorporated human-induced factors such as land fragmentation, urbanization, energy industrialization, and marine transportation. Even fewer have studied the scale effects. A study that captures and quantifies both natural and human factors as well as their neighborhood effects would help uncover the complex mechanism of land loss and provide a more accurate spatiotemporal projection of land loss patterns and probability.

The analysis procedures are as follows. (1) First, the study area is rasterized into 1-km by 1-km grids. (2) A set of natural and human variables related to land loss in the deltaic region are collected. (3) Variogram analysis of each variable is conducted to identify the spatial neighborhood size of each variable, and a neighborhood variable for each independent variable is created. (4) Elastic Net regression analysis is applied to test and select the significant variables that affect land loss. Regression results between the model with and the model without neighborhood variables are compared. Through this study, we should be able to derive a more accurate land loss model for detailed analysis and future projections. 